\title{
Fallstricke bei der Diabetesdiagnostik: Wird zu lax mit Laborwerten umgegangen?
}

\section{Pitfalls in the Diagnosis of Diabetes: Are we too Lax with Laboratory Parameters?}

Autoren

Rüdiger Landgraf ${ }^{1}$, Matthias Nauck², Guido Freckmann³, Ulrich A. Müller', Lutz Heinemann5, Monika Kellerer6, Dirk Müller-Wieland ${ }^{7}$

\section{(ㄷ) (1) $\circledast \circledast$}

Institute

1 Deutsche Diabetes Stiftung, München

2 Institut für Klinische Chemie und Laboratoriumsmedizin, Universitätsmedizin Greifswald, Greifswald

3 Institut für Diabetes-Technologie, Forschungs- und Entwicklungsgesellschaft mbH an der Universität Ulm, Ulm

4 Klinik für Innere Medizin III, Universitätsklinikum Jena, Jena

5 Science Consulting in Diabetes $\mathrm{GmbH}$, Neuss

6 Zentrum für Innere Medizin I, Marienhospital Stuttgart, Stuttgart

7 Medizinische Klinik I, RWTH-Aachen

Schlüsselwörter

Diabetesdiagnose, (prä)-analytische Probleme, Plasmaglukose, HbA1c, oGTT

Key words

diabetes diagnosis, (pre)-analytic problems, plasma glucose, HbA1c, oGTT

Bibliografie

DOI https://doi.org/10.1055/a-0673-2156

Online-Publikation: 20.9.2018

Dtsch Med Wochenschr 2018; 143: 1549-1555

(C) Georg Thieme Verlag KG, Stuttgart · New York ISSN 0012-0472

Korrespondenzadresse

Prof. Dr. med. Rüdiger Landgraf

Deutsche Diabetes Stiftung, Gaißacherstraße 18,

81371 München

ruediger.landgraf@gmx.de

\section{ZUSAMMENFASSUNG}

Die Diabetesdiagnose zeigt viele präanalytische und analytische Probleme. Nüchtern-Glukose (NG), orale Glukosetole- ranztest (oGTT) und HbA1c haben Vor- und Nachteile und sind nicht gleichwertig in ihrer diagnostischen Aussagekraft. Der oGTT ist der empfindlichste Test, die Reproduzierbarkeit ist relativ niedrig $(\mathrm{VK} \pm 15 \%)$. Die $\mathrm{NG}$ entdeckt maximal 70 $80 \%$ der Patienten mit einem manifesten Diabetes. Die Glukose-Messung wird durch unsachgemäße Blutabnahme, intraindividuelle Glukose-Schwankungen, Fastendauer und Fehler beim oGTT verfälscht. HbA1c ist trotz IFCC-Standardisierung bei erlaubter Abweichung von $\pm 18 \%$ in Ringversuchen und dem Einsatz von nicht kommutablem Ringversuchsmaterial schlecht überprüfbar. HbA1c-Analyse zeigt viele Interferenzen und ist nur begrenzt zur Diabetesdiagnose geeignet. Der Schwellenwert von $\mathrm{HbA} 1 \mathrm{c}$ von $\geq 6,5 \%(\geq 48 \mathrm{mmol} / \mathrm{mol} \mathrm{Hb})$ beruht nicht auf harter Evidenz. Der Aufwand bei der Diagnose (NG und/oder oGTT+HbA1c) ist bei den sich daraus ergebenden klinischen Konsequenzen minimal invasiv, zumutbar, preiswert und verhindert eine Über- und Unterdiagnostik.

\section{ABSTRACT}

The diagnosis of diabetes is associated with pre-analytical and analytical problems. Fasting glucose (FG), oral glucose tolerance test (oGTT) and HbA1c have advantages and shortcomings and have no equal diagnostic validity. oGTT is the most sensitive test, but its reproducibility is rather poor (CV $\pm 15 \%$ ). FG detects only $70-80 \%$ of overt diabetes. FG is falsified by inappropriate blood sampling, intra-individual fluctuations and mistakes with the oGTT. HbA1c despite IFC- standardization, but with a tolerable coefficient of variation of $\pm 18 \%$ in round robin tests and use of not commutable control material is not easy to interpret. HbA1c analysis shows also interferences and is therefore of limited diagnostic value. Its threshold value of $\geq 6.5 \%$ ( $\geq 48 \mathrm{mmol} / \mathrm{mol} \mathrm{Hb}$ ) is based on consensus and not on evidence. The diagnostic effort ( $F G$ and/or $\mathrm{oGTT}+\mathrm{HbA1c}$ ) with serious consequences is minimal invasive, reasonable and cheap. It prevents over- and underdiagnosis.
Der Diabetes mellitus ist eine chronische Krankheit mit zum Teil folgenreichen Komplikationen für den betroffenen Menschen sowie für sein soziales Umfeld, die Gesundheitsökonomie und die gesamte Volkswirtschaft. Die Diagnose basiert unabhängig von der Diabetes-Ursache auf einer zuverlässigen Labordiagnose, die einfach zu sein scheint, die aber eine Vielzahl von Fallstricken 
beinhaltet, die in der Praxis häufig ungenügend berücksichtigt werden.

\section{Methoden und Kriterien zur Diagnose eines Diabetes}

Anhand einer selektiven Literaturrecherche und unter Berücksichtigung nationaler und internationaler Leitlinien wurde dieser Review verfasst.

Diabetes-Symptome (typisch bei Typ-1-Diabetes mit akuter Stoffwechselentgleisung) oder ein erhöhtes Diabetes-Risiko (z. B. anhand von Fragebögen: Dife - Deutscher Diabetes-Risiko-Test ${ }^{\circledR}$ (DRT) [1] oder FINDRISK [2]) sollen zur Diabetesdiagnose führen:

1. Gelegenheits-Plasmaglukose $\geq 200 \mathrm{mg} / \mathrm{dl}(\geq 11,1 \mathrm{mmol} / \mathrm{l})$, insbesondere mit Diabetes-Symptomen oder

2. Nüchtern-Plasmaglukose $\geq 126 \mathrm{mg} / \mathrm{dl}(\geq 7,0 \mathrm{mmol} / \mathrm{l})$ oder

3. 2-Std. Plasmaglukose von $\geq 200(\geq 11,1 \mathrm{mmol} / \mathrm{l})$ nach $75 \mathrm{~g}$

Glukose in einem oralen Glukosetoleranztest (oGTT) oder

4. $\mathrm{HbA} 1 \mathrm{c} \geq 6,5 \%(\geq 48 \mathrm{mmol} / \mathrm{mol} \mathrm{Hb})$.

Diese Diagnosekriterien sind von der WHO [3], der International Diabetes Federation [4] und von vielen Diabetes-Fachgesellschaften übernommen worden [5 - 7].

Die Deutsche Diabetes Gesellschaft (DDG) zusammen mit der Deutschen Gesellschaft für Klinische Chemie und Laboratoriumsmedizin (DGKL) haben eine kritische Wertung der für die Diabetesdiagnose entscheidenden Laboranalysen vorgenommen, die für die Zuverlässigkeit der Diagnose ausschlaggebend sind und bisher nicht genügend thematisiert wurden.

\section{Diagnose-Prozedere}

Bei Messergebnissen der Laborparameter ist die Frage, ob die Abweichung vom diagnostischen Grenzwert so weit oberhalb dieses Grenzwertes liegt (d.h. größer ist als die Minimal Difference [s.u.]), dass dieser Laborwert mit Sicherheit erhöht ist. Dann würde eine Einzelbestimmung für die Diagnose-Stellung ausreichen, insbesondere von typischen Symptomen.

Grundsätzlich sollte aber die Diagnose auf der Basis von bestätigten Werten erfolgen.

Die Bestätigung kann erfolgen, indem derselbe Parameter zeitnah (z. B. innerhalb von 14 Tagen) in einer neuen Blutprobe analysiert wird oder indem ein anderer der oben aufgeführten vier Konstellationen herangezogen wird.

Wird ein Parameter verwendet und dieser zur Diagnosebestätigung wiederholt, muss davon ausgegangen werden, dass die Blutproben jeweils standardisiert „bearbeitet“ wurden. Dies ist insbesondere bei der Plasmaglukose nicht einfach überprüfbar und häufig nicht gewährleistet (s. u.).

Bei Grenzwerten wird eine engmaschige Betreuung des Patienten mit Wiederholung des diagnostischen Tests nach 3 - 6 Monaten empfohlen [5]. Bei divergenten Ergebnissen zwei diagnostischer Verfahren (oberhalb und unterhalb) des Grenzwertes sollte das Testverfahren mit dem Wert oberhalb des Grenzwertes wiederholt werden.
Erfolgt die Diabetesdiagnose mit einer HbA1c-Messung, dann ist die Bestätigungsmessung mit HbA1c nicht sinnvoll. Die HbA1c-Messung ist wegen einer Reihe analytischer Probleme unzureichend reproduzierbar [8] oder es wird erneut ein "falscher" Wert durch die gleichen patientenspezifischen Einflussgrößen erzeugt [9-13].

Unabhängig davon, welche Parameter zur Diagnosestellung verwendet werden, kann das Ergebnis durch patientenspezifische Einflussgrößen und/oder unzureichende Messgenauigkeit fehlerhaft sein.

\section{Plasmaglukose-Messung}

Die Glukose soll in venösem Plasma gemessen werden. Nicht selten wird jedoch in Vollblut (kapillar oder venös) oder im Serum gemessen.

\section{Präanalytische Probleme}

Diese sind praktisch sehr häufig, aber vermeidbar.

Nur die Verwendung geeigneter Blutentnahmeröhrchen (Natriumfluorid plus Citrat) hemmt die Glykolyse in vitro vollständig. Fluorid allein ist nicht ausreichend. Die aktuell am Markt befindlichen Blutentnahmeröhrchen mit Glykolyse-Inhibitoren weisen jedoch weitere Probleme auf, die in $>$ Tab. 1 aufgelistet sind. Röhrchen, die Granulat enthalten, sind etwas teurer, aber am besten geeignet. Nach der Blutentnahme muss zur GlykolyseHemmung das Röhrchen sofort intensiv (10-mal) gemischt werden. Bei den Röhrchen, die einen flüssigen Zusatz enthalten, muss das Röhrchen exakt mit Blut gefüllt werden. Es kommt jedoch häufig zu erheblichen Fehlern des Mischverhältnisses, die trotz des Einsatzes des Korrekturfaktors nicht ausgeglichen werden können.

Alternativ können Röhrchen ohne Zusatz zur sofortigen und vollständigen Glykolyse-Hemmung nach der Blutentnahme verwendet werden, wenn das Blut umgehend zentrifugiert wird. Pro Stunde erfolgt sonst in einem normalen Blutentnahmeröhrchen ein temperaturabhängiger anaerober Glukose-Abbau (ca. 0,5 mmol/Std.) mit dem Ergebnis falsch-niedriger Werte. Wird ein Zeitfenster von 30 Minuten bis zur Zentrifugation überschritten, sollten diese Proben wegen der ablaufenden Glykolyse verworfen werden. Nach der Zentrifugation muss der Plasmaüberstand von den Blutzellen unmittelbar getrennt werden. Die Sicherung der Diagnose erfolgt durch standardisierte und qualitätsgesicherte Laboruntersuchungen, die einen niedrigen Variationskoeffizienten der Messung haben (optimal: VK $1-3 \%)$. Diese Präzision ist bei den üblichen Point-of-CareTestsystemen (POCT) zur Selbstmessung der Glukose nicht gegeben (VK bis zu 15\%).

Kapillarblut stellt eine Mischung aus arteriellem und venösem Blut dar. Nüchtern liegt die Glukosekonzentration im venösen Blut um 2-10\% niedriger im Vergleich zu Kapillarblut [14, 15]. Postprandial steigt die kapillar-venöse Glukosedifferenz in Abhängigkeit von der oral aufgenommenen Kohlenhydratmenge und fällt nach 120 min wieder ab. Es gibt keine für die Diabetesdiagnose nutzbare Korrelation zwischen kapillaren und venösen Glukosewerten; somit soll nur venöses Plasma zur Glukosebestimmung verwendet werden. 
- Tab. 1 Blutentnahmeröhrchen speziell zur Bestimmung der Glukose (Quelle: Nauck M, Petermann A, Müller-Wieland D et al. Definition, Klassifikation und Diagnostik des Diabetes Mellitus. Diabetologie 2017; 12(2): S94-S100)

\begin{tabular}{|c|c|c|c|c|}
\hline Hersteller & Produktname & $\begin{array}{l}\text { korrekte Füllung } \\
\text { (absolut notwendig) }\end{array}$ & $\begin{array}{l}\text { ausreichendes } \\
\text { Mischen erforderlich }\end{array}$ & Korrekturfaktor \\
\hline Greiner bio-one & $\begin{array}{l}\text { Vacuette }{ }^{\circledR} \\
\text { FC-Mix }\end{array}$ & nein & 10-mal & nein (Granulat) \\
\hline Kabe & $\begin{array}{l}\text { Primavette }^{\circledR} \text {, } \\
\text { KABEVETTE }^{\circledR}\end{array}$ & ja & wenige Male & 1,16 (flüssiger Zusatz) \\
\hline Sarstedt & $\begin{array}{l}\text { S-Monovette } \\
\text { ClucoEXACT }{ }^{\circledR}\end{array}$ & ja & wenige Male & 1,16 (flüssiger Zusatz) \\
\hline \multicolumn{5}{|c|}{$\begin{array}{l}\text { Bei den Röhrchen der Firma Greiner bio-one (Vacuette }{ }^{\circledR} \text { FC-Mix) findet sich in den Blutentnahmeröhrchen ein Granulat. Die Röhrchen müssen nach der } \\
\text { Bluteinfüllung 10-mal geschwenkt werden, um eine ausreichende Lösung und Durchmischung mit dem Glykolysehemmer zu erreichen. } \\
\text { Bei den Blutentnahmeröhrchen der Firma Sarstedt (S-Monovette GlucoEXACT }{ }^{\circledR} \text { ) und der Firma Kabe (Primavette }{ }^{\circledR}, \text { KABEVETTE }^{\circledR} \text { ) zeigt die Erfahrung, dass } \\
\text { es durch das nicht vollständige Füllen der Röhrchen zu Verdünnungsfehlern kommt. Das Labor muss solche Röhrchen sicher identifizieren, um einerseits } \\
\text { die Röhrchen erkennen zu können, die nicht entsprechend den Vorgaben der Hersteller korrekt gefüllt sind und diese von der Analyse auszuschließen und } \\
\text { um bei korrekter Füllung den Verdünnungsfaktor von } 1,16 \text { zu berücksichtigen. }\end{array}$} \\
\hline
\end{tabular}

\section{Analytische Probleme}

Die enzymatischen Testsysteme zur spezifischen Messung der Glukose sind etabliert. Dennoch ist nach den Richtlinien der Bundesärztekammer zur Qualitätssicherung labormedizinischer Untersuchungen (Rili-BÄK) eine zulässige relative Abweichung des Einzelwertes für Glukose von $\pm 11 \%$ (interne Qualitätskontrolle) und eine zulässige relative Abweichung beim Ringversuch von $\pm 15 \%$ (externe Qualitätskontrolle) erlaubt [16]. Diese Abweichungen sind zur Diabetesdiagnose nicht akzeptabel, zumal die meisten Laboratorien wesentlich genauer analysieren.

\section{Merke}

Der In-vitro-Glukoseabbau kann nach venöser Blutentnahme weitgehend verhindert werden:

- vollständige Glykolyse-Hemmung durch Verwendung von Blutentnahme-Röhrchen mit Fluorid plus Zitrat-Zusatz

- Plasmaglukose sofort messen

- Blutröhrchen innerhalb von <30 min zentrifugieren und Plasma separieren

\section{Nüchtern-Glukose}

Der Glukose-Assay ist spezifisch, einfach durchführbar, überall verfügbar (meist automatisiert) und preiswert.

Es gibt jedoch eine Reihe von Problemen:

- intra-individuelle biologische Schwankung von Tag zu Tag $( \pm 5-14 \%)[17,18]$

- Unsicherheit des Nüchtern-Zustandes der zu testenden Person. Der Patient muss 8 - 12 Stunden sicher nüchtern sein.

- Tageszeitliche Schwankung. Testung daher am Morgen zwischen 8 und 9 Uhr

- Eine Reihe von Faktoren beeinflussen akut die Plasmaglukosekonzentration (z. B. akute Infektionen, Stress, Steroide ...).

- Die Nüchtern-Glukose ist wenig sensitiv, um einen Diabetes im Frühstadium zu entdecken. Bei etwa einem Drittel von Menschen mit Diabetes manifestiert sich die Krankheit als post- prandiale Hyperglykämie [19] und entzieht sich somit einer Frühdiagnose bei Verwendung von Nüchtern-Glukosewerten.

\section{Minimal Difference (MD) und Interpretation des Messwertes}

Alle Labormessungen sind grundsätzlich mit Fehlern behaftet, dessen Ausmaß der Anwender ohne zusätzliche Informationen nicht beurteilen kann [20]. Die Minimal Difference (MD) ist ein einfacher Parameter, der dem Anwender die Bedeutung des zufälligen Fehlers von Laborwerten veranschaulicht.

Der zufällige Fehler wird quantitativ erfasst, indem die Streuung von wiederholten Messungen beschrieben und als Variationskoeffizient (VK) angegeben wird.

Der VK berechnet sich aus Standardabweichung (SD) und Mittelwert (MW) und wird in Prozent angegeben: $\mathrm{VK}=\mathrm{SD}$ mal 100/MW.

Die Minimal Difference (MD) gibt konkrete Konzentrationen in absoluten Werten an, d. h. ab wann sich ein Messwert mit einem Vertrauensbereich von $95 \%$ von einem Grenzwert unterscheidet.

Die MD berechnet sich nach folgender Formel: MD = 2-mal SD.

Bei einem Grenzwert von $100 \mathrm{mg} / \mathrm{dl}(5,6 \mathrm{mmol} / \mathrm{l})$ beträgt die MD abhängig vom VK: $\pm 4 \mathrm{mg} / \mathrm{dl}(0,2 \mathrm{mmol} / \mathrm{l})$ für einen VK von $2 \%$ und $\pm 10 \mathrm{mg} / \mathrm{dl}(0,6 \mathrm{mmol} / \mathrm{l})$ bei einem VK von $5 \%$. Das heißt, dass sich ein Glukosemesswert von einem Grenzwert von $100 \mathrm{mg} / \mathrm{dl}(5,6 \mathrm{mmol} / \mathrm{l})$ analytisch dann unterscheidet, wenn er bei einem VK von $2 \%:<96 \mathrm{mg} / \mathrm{dl}(<5,3 \mathrm{mmol} / \mathrm{l})$ bzw. $>104 \mathrm{mg} / \mathrm{dl}$ $(>5,8 \mathrm{mmol} / \mathrm{l})$ ist. Bei einem VK von $5 \%$ liegen diese Werte bei $<90 \mathrm{mg} / \mathrm{dl}(<5,0 \mathrm{mmol} / \mathrm{l}) \mathrm{bzw} .>110 \mathrm{mg} / \mathrm{dl}(>6,1 \mathrm{mmol} / \mathrm{l})$. Diese Streuung hat daher eine erhebliche Bedeutung für die Diagnosestellung eines Diabetes. Um die Auswirkungen des zufälligen Fehlers zu verdeutlichen, hat die Kommission Labordiagnostik in der Diabetologie (KLD) der DDG und der DGKL empfohlen, die Information zur MD des entsprechenden Laboratoriums für die Analyte Glukose und HbA1c für wichtige Cut-Off-Werte routinemäßig anzugeben [7, 20]. 
- Tab. 2 Durchführung des oGTT (75 g Glukose) nach WHO-Richtlinien.

$$
\begin{aligned}
& \text { Voraussetzung } \\
& \text { Testdurchführung am Morgen }
\end{aligned}
$$

zum Zeitpunkt 0 Trinken von $75 \mathrm{~g}$ Glukose (oder äquivalenter Menge hydrolysierter Stärke) in 250 - $300 \mathrm{ml}$ Wasser; innerhalb von 5 Min. trinken

Test kontraindiziert bei:

\section{Erläuterung}

- nach 8 - 12 Stunden Nahrungs-, Nikotin- und Alkohol-Karenz

- nach einer $\geq 3$-tägigen kohlenhydratreichen Ernährung ( $\geq 150 \mathrm{~g} \mathrm{KH}$ pro Tag)

- im Sitzen oder Liegen (keine Muskelanstrengung); nicht rauchen vor oder während des Tests

- Kinder 1,75 g/kg Körpergewicht (max. $75 \mathrm{~g}$ )

- Blutentnahme zu den Zeitpunkten 0 und 120 Min.

- sachgerechte Probenentnahme, -verarbeitung und -aufbewahrung

- interkurrenten Erkrankungen

- vorübergehende Einnahme diabetogener Medikamente

- Stressbedingungen wie kurz nach invasiven Eingriffen, Myokardinfarkt oder Schlaganfall

- bei Z. n. Magen-Darm-Resektion oder gastrointestinalen Erkrankungen mit veränderter Resorption

- bei bereits manifestem Diabetes

\section{Oraler Glukosetoleranztest (oGTT; $75 \mathrm{~g}$ )}

Der oGTT misst den Glukoseanstieg und die Eliminationsrate der Glukose aus dem Blut. Diese wird beeinflusst von der Schnelligkeit der Magenentleerung, der intestinalen Glukoseaufnahme ins Blut, der adäquaten Freisetzung von Insulin und dem Grad der Insulinresistenz. Weiteren Faktoren wie mangelnde Standardisierung des Tests und Krankheiten (z. B. Magenerkrankungen, Medikamenten, Drogen wie Alkohol) können neben einer physiologischen Varianz das Ergebnis des oGTT beeinflussen.

Der Test wird von allen wichtigen Fachgesellschaften weltweit einschließlich WHO und International Diabetes Federation (IDF) empfohlen und ist keineswegs wissenschaftlich umstritten, sondern bei korrekter Durchführung und Indikation unverzichtbar für die Hyperglykämie-Diagnostik. Die Fachgesellschaften sind sich bewusst, dass der oGTT eine Reihe von Problemen aufweist, wie z. B. geringere Praktikabilität, etwas höhere Kosten und eine mäßigere Reproduzierbarkeit (bis zu $\pm 15 \%$; [21-27]) als die Nüchtern-Glukose und das HbA1c (s.u.). Die Sensitivität zur Diabetes-Diagnostik ist jedoch höher als die der NüchternGlukose und des HbA1c. Die Reproduzierbarkeit des 2-Std.-Wertes im oGTT kann jedoch deutlich erhöht werden, wenn der Test strikt standardisiert durchgeführt wird ( $\vee$ Tab. 2), die Probenentnahme und -verarbeitung für die Glukoseanalyse den diskutierten Richtlinien entspricht (s. o.) und Kontraindikationen beachtet werden. Wichtig ist, dass die Blutprobe für den 2-Std.-Wert exakt nach 120 Minuten ( \pm 5 Min.) abgenommen wird.

Die seit Jahrzehnten eingeführte und bewährte Fertiglösung (z. B. Dextro O.G.T. ${ }^{\circledR}$ ) sollte nicht aus Kostengründen durch eine selbstgemischte Glukoselösung ersetzt werden, weil:

- sich Glukose-Monohydrat relativ schlecht in Wasser löst und damit die Fertigstellung zeitaufwändig ist und Fehler bei der kompletten Lösung der exakten Menge (!) in dem vorgegebenen Volumen (!) vorprogrammiert sind

- damit wesentliche Gründe für eine schlechtere Reproduzierbarkeit der oGTT bestehen und eine exakte Diagnose des Diabetes in Frage gestellt ist
- die Bezahlung für Abwiegen und Verpackung der Glukose durch den Apotheker nicht geregelt ist

- der Aufwand in der Praxis in keinem Verhältnis zu den Kostenersparnissen steht (wurde nie offiziell berechnet!)

- Die Fertigstellung der Lösung durch den Arzt selbst, anstatt durch den Hersteller, wird von der DDG aus haftungsrechtlichen und medizinischen Gründen nicht empfohlen.

\section{Merke}

Zahlreiche Studien haben gezeigt, dass die alternativen Kriterien zur Diabetesdiagnose, also Fastenplasmaglukose oder 2-Std.-Wert nach oGTT oder HbA1c, nicht die gleichen Personen als Diabetespatienten identifizieren [28 - 32], was zur Überoder Unterdiagnostik führt und epidemiologische Aussagen zumindest unscharf macht.

\section{HbA1c zur Diagnose einer chronischen Hyperglykämie}

Die Diagnostik eines Diabetes mithilfe des glykierten Hämoglobins (HbA1c) hat relevante Vorteile:

\section{INFO}

Vorteile von HbA1c-Bestimmungen zur Diabetesdiagnostik

- weniger anfällig für präanalytische Fehler im Vergleich zur Plasmaglukose

- geringere intraindividuelle biologische Variabilität

- vernachlässigbare tageszeitliche Schwankungen

- nüchtern: keine Voraussetzung für die Bestimmung

- geringer Einfluss durch akuten Stress (z. B. Infektionen)

- HbA1c reflektiert die mittlere Plasmaglukose der letzten 8-12 Wochen (biostatistisch!).

- bequemer für Patient und Arzt im Vergleich zum oGTT

- billiger in der Analytik als ein oGTT 
- Tab. 3 Faktoren, die zu einer Beeinflussung oder Verfälschung des HbA1c-Messwertes führen [10 - 12].

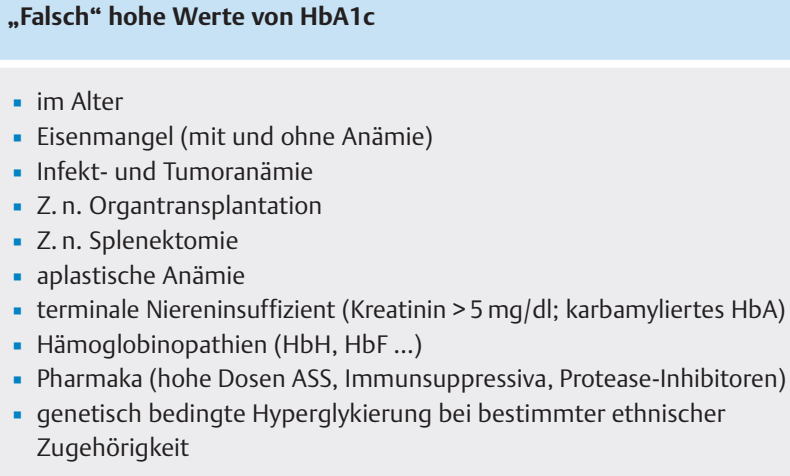

\section{„Falsch“ niedrige Werte von HbA1c}

- große Höhen (>3000 m)

- Folsäuremangel (Schwangerschaft)

- hämolytische Anämie

- Blutverlust

- nach Bluttransfusionen

- Pharmaka (Erythropoetin, Eisen-supplementierung)

- verkürztes Erythrozyten-Überleben bei

- Niereninsuffizienz, Leberzirrhose

- ernährungsbedingt (fettreich, Alkohol)

- Hämoglobinopathien (HbS, HbC, HbD ...)
Aus diesen Gründen haben sich Fachgesellschaften wie die ADA u. a., sowie Institutionen wie IDF und WHO beginnend 2010 entschlossen, HbA1c als Diagnoseparameter einzuführen [3-7]. Die Standardisierung der HbA1c-Assays ist zwar verpflichtend, jedoch zumindest in Deutschland noch unzureichend umgesetzt [9].

Die HbA1c-Bestimmung ist trotz Verwendung des IFCC- Standards nicht ausreichend sensitiv und in vielen Regionen nicht verfügbar [5]. Kürzlich konnte gezeigt werden, dass der mittlere IFCC standardisierte HbA1c-Wert bei normalen, mäßig und stark erhöhten HbA1c-Werten um absolut 0,5\% (5,4 mmol/mol Hb) zwischen 4 zertifizierten deutschen Laboratorien differiert [33]. Dies ist kann zu Fehldiagnosen und -entscheidungen in der Therapie führen. Nach Rili-BÄK ist zudem eine zulässige interne Qualitätskontrolle von $\pm 10 \%$ und eine zulässige externe Qualitätskontrolle beim Ringversuch von $\pm 18 \%$ erlaubt [16]. Dies bedeutet, dass ein Sollwert von $48 \mathrm{mmol} / \mathrm{mol} \mathrm{Hb}$ (6,5\%) bei der internen Qualitätskontrolle 43,2 - 52,8 mmol/mol Hb (5,8 - 7,2\%) und bei der externen Qualitätskontrolle 39,4 - 56,6 mmol/mol Hb (5,3-7,8\%) betragen kann. Daher fordert die Kommission für Labordiagnostik in der Diabetologie (KLD) der DDG/DGKL zur Diagnose- und Therapiesicherheit von HbA1c striktere Grenzen für die interne $( \pm 3 \%)$ und die externe $( \pm 8 \%)$ Qualitätskontrolle einzuführen. Dies gelingt durch Verwendung von kommutablem Ringversuchsmaterial (Frischblut) bei den Ringversuchen [9]. Richtlinien für die externe Qualitätskontrolle für HbA1c-Messung liegen in Ländern wie USA, Niederlande, England, Schweiz bei $\pm 6 \%$ und in China bei $\pm 8 \%$.

Die Analyse und Interpretation des HbA1c hat zusätzlich eine Reihe von Störfaktoren und ist daher nur begrenzt als Instrument zur Diabetesdiagnose geeignet ( $\triangleright$ Tab. 3 ).

Insbesondere der Anstieg des HbA1c um absolut 0,5\% $(5,4 \mathrm{mmol} / \mathrm{mol} \mathrm{Hb})$ im Alter von 30 auf $>70$ Jahre kann zu einer Überdiagnostik des Diabetes im Alter führen [34].

Neben der Berücksichtigung möglicher Diskrepanzen zwischen Plasmaglukosewerten und $\mathrm{HbA} 1 \mathrm{c}$ sollte der HbA1c bei folgenden Menschen mit veränderter Erythrozyten-Lebensdauer und bei Frischmanifestation eines Diabetes nicht zur Diagnose eingesetzt werden:

\section{INFO}

HbA1c ist zur Diabetes-Diagnose bei folgenden Situationen ungeeignet

- Kinder und Jugendliche (umstritten, da $\geq 6,5 \%$ [ $\geq 48 \mathrm{mmol} / \mathrm{mol} \mathrm{Hb}$ ] zu hoch)

- Diagnostik eines Schwangerschaftsdiabetes

- Schwangere bis 3 Monate postpartum

- Menschen mit Verdacht auf Typ-1-Diabetes (jedes Alter)

- Menschen mit akuten Diabetes-Symptomen

- Prädiabetes mit akuter Stresshyperglykämie (Apoplexie, Myokardinfarkt, Trauma etc.)

- Menschen mit Medikamenten (<ca. 2 Monate), die zu einem Blutglukoseanstieg führen (z. B. Glukokortikoide, Psychopharmaka, Protease-Inhibitoren)

- Z. n. Pankreasoperation, akute Pankreaserkrankung

- HIV-Patienten

- progrediente Niereninsuffizienz

Die Festlegung des Schwellenwerts von einem HbA1c ist sehr umstritten, denn in dem Bereich des HbA1c von $\geq 6,5 \%$ ( $\geq 48 \mathrm{mmol} /$ $\mathrm{mol} \mathrm{Hb}$ ) ist die Spezifität des Testes zwar hoch, aber die Sensitivität nur $<50 \%$. Somit werden nach den international akzeptierten Plasmaglukose-Diagnosekriterien weniger als $50 \%$ der untersuchten Personen als manifeste Diabetiker eingestuft [36-38]. HbA1c zur Frühdiagnose eines Diabetes heranzuziehen macht bei dem genannten Schwellenwert auch wenig Sinn, wenn die Diagnose an bereits klinisch erkennbaren Komplikationen wie der diabetesspezifischen Retinopathie festgemacht wird.

Daher fordert die DDG/DGIM und DGKL in einem Bereich des HbA1c von $>5,7$ bis < 6,5\% zusätzlich Plasmaglukose-Parameter (Nüchtern-Glukose und/oder 2-Std.-Wert nach oGTT) zur Diagnostik heranzuziehen [7].

In der kürzlich erschienenen Metaanalyse von Hoyer et al. [39] fand sich ein sinnvoller Schwellenwert für die Diagnose eines Diabetes auf der Basis eines 2-Std.-Plasmaglukosewertes von $\geq 200 \mathrm{mg} / \mathrm{dl}$ ( $\geq 11,1 \mathrm{mmol} / \mathrm{l})$ für $\mathrm{HbA} 1 \mathrm{c}$ bei 42 und $44 \mathrm{mmol} / \mathrm{mol}$ Hb (6,0 - 6,2\%) und für die Nüchtern-Plasmaglukose bei 112 $115 \mathrm{mg} / \mathrm{dl}(6,2-6,4 \mathrm{mmol} / \mathrm{l})$ mit einer Sensitivität von 68,4\% 
(95\% Cl:46,6 - 84,3\%) und 68,7\% (95\% Cl: 50,6 - 82,4\%), sowie eine Spezifität von 95,9\% (95\% Cl: 85,4-98,9\%) und 93,7\% (95\% Cl: 78,0 - 98,4\%) für HbA1c bzw. Nüchtern-Plasmaglukose. Dass zur Diagnose eines Diabetes inkorrekt der Cut-off des HbA1c bei $\geq 48 \mathrm{mmol} / \mathrm{mol} \mathrm{Hb}$ ( $\geq 6,5 \%$ ) festgelegt wurde, zeigt auch eine aktuelle Studie aus Schweden und Finnland mit 4 unterschiedlichen Kohorten [40]. Mit einem HbA1c-Wert von 6,5\% wurden nur $5 \%$ der Menschen entdeckt, die auf dem Boden von Glukose-Kriterien (Nüchtern-Plasmaglukose und/oder 2-Std.Wert nach oGTT) bereits als Personen mit Diabetes identifiziert wurden. Der konsentierte Diagnose-Algorithmus der KLD trägt dieser Problematik Rechnung [7].

\section{KERNAUSSAGEN}

- Präanalytische Probleme bei der Glukosebestimmung können durch sachgerechte Probenentnahme (Fluorid+ZitratRöhrchen), -verarbeitung und -aufbewahrung vermieden werden.

- Die Berücksichtigung des zufälligen Analysefehlers (Minimal Difference) für Glukose und HbA1c erhöht die Diagnosesicherheit.

- Nüchtern-Glukose erkennt nur in ca. 70 \% einen manifesten Diabetes.

- Der oGTT ist der sensitivste Test zur Erkennung eines Diabetes.

- Die diagnostische Wertigkeit von HbA1c ist durch Präzision der Analyse und mögliche Beeinflussung oder Verfälschung der Werte limitiert. Die Kombination aus Plasmaglukose (nüchtern und/oder oGTT) und HbA1c ist sinnvoll, minimal invasiv und verhindert eine Über- und Unterdiagnostik des Diabetes.

Interessenkonflikt

R. Landgraf erklärt potenzielle Interessenkonflikte. Advisory Boards: Lilly Deutschland, Novo Nordisk Pharma; Vortragshonorare: Bayer Vital, Berlin Chemie, Lilly Deutschland, Novo Nordisk Pharma. Andere Aktivitäten: Bevollmächtigter des Vorstands der Deutschen Diabetes Stiftung, Steuerungsgruppe für die Entwicklung und Aktualisierung der Nationalen Versorgungs-Leitlinien Diabetes

G. Freckmann hat als Leiter des Instituts für Diabetes-Technologie Forschungs- und Entwicklungsgesellschaft an der Universität Ulm erklärt folgende potenzielle Interessenkonflikte: Durchführung klinischer Studien auf Eigeninitiative und für verschiedene Medizinproduktefirmen. Er erhält Vortrags-Beratungshonorare von: Abbott, Ascensia, Novo Nordisk, Roche, Sanofi, Sensile, Ypsomed

L. Heinemann erklärt potenzielle Interessenkonflikte: Advisory Boards: Abbott, Lifecare, Novo Nordisk, Deutschland, Roche Diagnostics, Sanofi, Unomedical. Berater für Ascensia, CellNovo, Heraeus, Insulet, Metronom, Roche Diagnostics. Vortragshonorare: Ascensia, Berlin-Chemie, Lilly Deutschland, Roche Diagnostics, Sanofi

M. Kellerer erklärt als Mitautorin folgende potenzielle Interessenkonflikte: Forschungsunterstützung: GlaxoSmithKline, AstraZeneca; Beratertätigkeit: Abbott, AstraZeneca, Boehringer Ingelheim, Lilly, MSD, Novo Nordisk, Vortragstätigkeit: AstraZeneca, Boehringer Ingelheim, Lilly, MSD, Novo Nordisk, Novartis, Janssen-Cilag, Sanofi UA. Müller hat seit 2010 keine persönlichen Honorare oder Reisekosten von pharmazeutischen Unternehmen erhalten. Seine Arbeitsgruppe erhielt Forschungsunterstützung von Fresenius Medical Care, VDBD, Diabeteszentrum Thüringen e. V., Haemopharm, NOVO Nordisk, Abbott, Pfizer Pharma, European Association for the Study of Diabetes auf ein Drittmittelkonto des Universitätsklinikums Jena

D. Müller-Wieland erklärt als Mitautor folgende potenzielle Interessenkonflikte. Mitglied in Advisory Board und Vortragshonorare in den letzten 3 Jahren von folgenden Firmen: Amgen, MSD, AstraZeneca, Novartis, Boehringer Ingelheim, Lilly, Novo Nordisk, Roche Diabetes Care, Sanofi M. Nauck erklärt folgende potenzielle Interessenkonflikte. Advisory Boards: Becton Dickinson. Vortragshonorare: Boehringer Ingelheim, Roche Diagnostics, Siemens; Ringversuchsleiter bei INSTAND e. V.; Mitglied des Präsidiums der DGKL und des Stiftungsbeirats des RfB; Berufung von der BÄK zum Mitglied des Beirats und von D1 zur Richtlinie der Bundesärztekammer zur Qualitätssicherung laboratoriumsmedizinischer Untersuchungen

\section{Literatur}

[1] Schulze M. Dife - Deutscher Diabetes-Risiko-Test (DRT). Diabetologie 2017; 12 (2): S264 - S267

[2] Schwarz P. FINDRISK-Test. Diabetologie 2017; 12 (2): S268 - S270

[3] WHO. Definition and diagnosis of diabetes mellitus and intermediate hyperglycemia. Report of a WHO/IDF Consultation; World Health Organization; 2006.; ISBN 9789241594936

[4] International Diabetes Federation. IDF clinical practice recommendations for managing type 2 diabetes in primary care. 2017

[5] American Diabetes Association. Classification and diagnosis of diabetes: standards of medical care in diabetes-2018. Diabetes Care 2018; 41 (1): S13-S27

[6] The International Expert Committee. International Expert Committee Report on the Role of the A1C Assay in the Diagnosis of Diabetes. Diabetes Care 2009; 32 (7): 1327-1334

[7] Nauck M, Petermann A, Müller-Wieland D et al. Definition, Klassifikation und Diagnostik des Diabetes Mellitus. Diabetologie 2017; 12 (2): S94S100

[8] Kerner W, Freckmann G, Müller UA et al. Positionspapier der Kommission für Labordiagnostik in der Diabetologie der DGKL und der DDG. Diabetologie 2015; 10: 329-333

[9] Heinemann L, Kaiser P, Freckmann G et al. HbA1c-Messung in Deutschland: Ist die Qualität ausreichend für Verlaufskontrolle und Diagnose? Positionspapier der Kommission für Labordiagnostik in der Diabetologie der DGKL und der DDG. Diabetologie 2018; 13: 46-53

[10] Sacks DB. A1C versus glucose testing. A comparison. Diabetes Care 2011; 34: 518-523

[11] Kowall B, Rathmann W, Landgraf R. Is HbA1c a valid and feasible tool for the diagnosis of diabetes? Diabetes Res Clin Pract 2011; 93: 314-316

[12] Dagogo-Jack S. Pitfalls in the use of HbA1c as a diagnostic test: the ethnic conundrum. Nat Rev Endocrinol 2010; 6: 589-593

[13] Bonora E, Tuomiletho J. The pros and cons of diagnosing diabetes with A1C. Diabetes Care 2011; 34 (2): S184-S190

[14] Larsson-Cohn U. Differences between capillary and venous blood glucose during oral glucose tolerance tests. Scand J Clin Lab Invest 1976; 36: $805-808$

[15] Irjala K, Koskinen P, Näntö $\vee$ et al. Interpretation of oral glucose tolerance test: Capillary-venous difference in blood glucose and the effect of analytical method. Scand J Clin Lab Invest 1986; 46: 307-313

[16] Bundesärztekammer. Neufassung der „Richtlinie der Bundesärztekammer zur Qualitätssicherung laboratoriumsmedizinischer Untersuchungen - Rili-BÄK. Dtsch Ärztebl 2014; 111 (38): A1583-A1618

[17] Selvin E, Crainiceanu CM, Brancati FL et al. Short-term variability in measures of glycemia and implications for the classification of diabetes. Arch Intern Med 2007; 167 (14): 1545-1551 
[18] Ollerton RL, Playle R, Ahmed K et al. Day-to-day variability of fasting plasma glucose in newly diagnosed type 2 diabetic subjects. Diabetes Care 1999; 22: $394-398$

[19] The DECODE-study group on behalf of the European Diabetes Epidemiology Group. Is fasting glucose sufficient to define diabetes? Epidemiological data from 20 European studies. Diabetologia 1999; 42: $647-654$

[20] www.deutsche-diabetes-gesellschaft.de/fileadmin/Redakteur/Stellung nahmen/2017/DDG_Stellungnahme_MD.pdf

[21] Köbberling J, Kerlin A, Creutzfeldt W. The reproducibility of the oral glucose tolerance test over long (5 years) and short periods ( 1 week). Klin Wochenschr 1980; 58: 527-530

[22] Ko GTC, Chan JCN, Woo J et al. The reproducibility and usefulness of the oral glucose tolerance test in screening for diabetes and other cardiovascular risk factors. Ann Clin Biochem 1998; 35: 62-67

[23] Gordon BA, Fraser SF, Bird SR et al. Reproducibility of multiple repeated oral glucose tolerance tests. Diabetes Res Clin Pract 2011; 94: e78 - e82

[24] Ye Y, Xie H, Zhao X et al. The oral glucose tolerance test for the diagnosis of diabetes mellitus in patients during acute coronary syndrome hospitalization: a meta-analysis of diagnostic test accuracy. Cardiovascular Diabetology 2012; 11: 155-165

[25] Van de Velde FP, Dierickx A, Depypere H. Reproducibility and least significant differences of oral glucose tolerance test-derived parameters in a postmenopausal population without diabetes. Diabetes \& Metabolism 2017; 43: $484-487$

[26] Chai JH, Ma S, Heng D. Impact of analytical and biological variations on classification of diabetes using fasting plasma glucose, oral glucose tolerance test and HbA1c. Sci Rep 2017; 7 (1): 13721

[27] Menke A, Rust KF, Cowie CC. Diabetes based on 2-h plasma glucose among those classified as having prediabetes based on fasting plasma glucose or A1c. Diab Vasc Dis Res 2018; 15 (1): 46 - 54

[28] Cowie CC, Rust KF, Byrd-Holt DD et al. Prevalence of diabetes and high risk for diabetes using A1c Criteria in the U.S. population in 1988-2006. Diabetes Care 2010; 33: 562-568

[29] van 't Riet E, Alssema M, Rijkelijkhuizen JM et al. Relationship between A1C and glucose levels in the general dutch population. The New Hoorn Study. Diabetes Care 2010; 33 (1): 61-66
[30] Fajans SS, Herman WH, Oral EA. Insufficient sensitivity of hemoglobin A1C determination in diagnosis or screening of early diabetic states. Metabolism Clin Exp 2011; 60: 86-91

[31] Gyberg V, De Bacquer D, Kotseva K et al. behalf of EUROASPIRE IV Investigators. Screening for dysglycaemia in patients with coronary artery disease as reflected by fasting glucose, oral glucose tolerance test, and HbA1c: a report from EUROASPIRE IV-a survey from the European Society of Cardiology. Eur Heart J 2015; 36: 1171-1177

[32] Shahim B, Gyberg V, De Bacquer D et al. Undetected dysglycaemia common in primary care patients treated for hypertension and/or dyslipidaemia: on the need for a screening strategy in clinical practice. A report from EUROASPIRE IV a registry from the Euro Observational Research Programme of the European Society of Cardiology. Cardiovasc Diabetol 2018; 17: $21-32$

[33] Roth J, Müller N, Lehmann T et al. Comparison of HbA1c measurements using 3 methods in 75 patients referred to one outpatient department. Exp Clin Endocrinol Diabetes 2018; 126 (1): 23 -26

[34] Roth J, Müller N, Lehmann T et al. HbA1c and age in non-diabetic subjects: An ignored association? Exp Clin Endocrinol Diabetes 2016; 124 (10): 637-642

[35] Colagiuri S, Lee CM, Wong TY et al. for the DETECT-2 Collaboration Writing Group. Glycemic thresholds for diabetes-specific retinopathy: implications for diagnostic criteria for diabetes. Diabetes Care 2011; 34 (1): $145-150$

[36] Peter A, Fritsche A, Stefan N et al. Diagnostic value of hemoglobin A1c for type 2 diabetes mellitus in a population at risk. Exp Clin Endocrinol Diabetes 2011; 119 (4): 234-237

[37] Selvin E, Steffes MW, Gregg E. Performance of A1C for the classification and prediction of diabetes. Diabetes Care 2011; 34: 84-89

[38] Kowall B, Rathmann W. HbA1c for diagnosis of type 2 diabetes. Is there an optimal cut point to assess high risk of diabetes complications, and how well does the $6.5 \%$ cutoff perform? Diabetes, Metabolic Syndr Obesity: Targets and Therapy 2013; 6: 477-491

[39] Hoyer A, Rathmann W, Kuss O. Utility of HbA1c and fasting plasma glucose for screening of Type 2 diabetes: a meta-analysis of full ROC curves. Diabet Med 2018; 35: 317-322

[40] Hellgren M, Steiner KH, Bennet L. Haemoglobin A1c as a screening tool for type 2 diabetes and prediabetes in populations of Swedish and Middle-East ancestry. Primary Care Diabetes 2017; 11: 337-343 